\title{
Angiorressonância magnética intracraniana 3D "time-of-flight" (TOF)
}

\author{
Claudia da Costa Leite ${ }^{1}$
}

A presença de fluxo no interior de um vaso que está sujeito a um campo magnético e ondas de radiofreqüência produz alterações de sinal que possibilitam a distinção entre os vasos sanguíneos e os tecidos estacionários ao seu redor.

A técnica de angiorressonância conhecida como 3D "time-of-flight" (tempo de vôo) promove contraste entre as estruturas vasculares com fluxo e o tecido estacionário numa única aquisição. Existem duas maneiras desta seqüência ser realizada: sem e com o uso de contraste paramagnético (gadolínio).

Em relação à angiografia digital, a angiorressonância (angio-RM) com a técnica 3D-TOF tem como vantagens o fato de não ser invasiva, de não utilizar radiação ionizante ou contraste iodado. Entre as desvantagens desta técnica de angio-RM devem ser destacadas a impossibilidade de estudar todo o segmento intracraniano em uma única aquisição, a dificuldade de detecção de vasos de pequeno calibre (em especial na seqüência 3D-TOF sem contraste paramagnético) e a possibilidade de um trombo com meta-hemoglobina simular um vaso com fluxo. Trombos ou hematomas contendo meta-hemoglobina não são saturados como o tecido estacionário, pois apresentam T1 mais curto do que os outros tecidos.

A angio-RM avalia as estruturas vasculares intracranianas, podendo ser realizada com ênfase no sistema arterial ou venoso. A técnica 3D-TOF com gadolínio é muito utilizada para o sistema venoso. Quando utilizada no estudo do sistema arterial, possibilita uma melhor identificação de ramos distais com menor calibre do que na técnica 3D-TOF sem contraste, porém há maior "conta-

1. Professora Livre-Docente do Departamento de Radiologia da Faculdade de Medicina da Universidade de São Paulo (FMUSP), Chefe do Setor de Ressonância Magnética do Hospital das Clínicas da FMUSP. minação" com as estruturas venosas. Variações anatômicas devem ser conhecidas, pois é possível identificá-las.

Muitas doenças vasculares do sistema nervoso central são diagnosticadas com o uso da ressonância magnética, sendo que a angio-RM já é utilizada desde o início da década passada. Sendo uma técnica de obtenção de imagens angiográficas de maneira segura e acurada, esta é rotineiramente utilizada na avaliação de pacientes com doença arterial vaso-oclusiva, aneurismas, trombose venosa, entre outros. Para aneurismas há trabalhos que relatam sensibilidade de $85 \%$ a $95 \%$ em aneurismas rompidos ou maiores que $5 \mathrm{~mm}$, mas há trabalhos com boa sensibilidade para detecção de aneurismas de até $3 \mathrm{~mm}$.

O trabalho publicado neste número da Radiologia Brasileira incluiu uma casuística de 100 pacientes que realizaram angio-RM devido a diferentes indicações clínicas, especialmente cefaléia, acidente vascular cerebral e ataque isquêmico transitório. Este trabalho tem aplicação na prática clínica, tendo demonstrado alteração em $52 \%$ dos exames realizados, sendo os principais achados a estenose vascular e o aneurisma. Apresenta uma análise crítica dos achados de angio-RM e da utilização desta técnica nos vasos intracranianos.

\section{REFERÊNCIAS}

1. Amoli SR, Turski PA. The role of MR angiography in the evaluation of acute stroke. Neuroimaging Clin N Am 1999;9:423-38.

2. Anzalone N, Scomazzoni F, Strada L, Patay Z, Scotti G. Intracranial vascular malformations. Eur Radiol 1998;8:685-90.

3. Edelman RR. Basic principles of magnetic resonance angiography. Cardiovasc Intervent Radiol 1992;15:3-13.

4. Summers PE, Jarosz JM, Markus H. MR Angiography in cerebrovascular disease. Clin Radiol 2001;56:437-56.

5. Uchino A, Kato A, Takase Y, Kudo S. Persistent trigeminal artery variants detected by MR angiography. Eur Radiol 2000;10: 1801-4.

6. Yang JJ, Hill MD, Morrish WF, et al. Comparison of pre- and postcontrast 3D time-of-flight MR angiography for the evaluation of distal intracranial branch occlusions in acute ischemic stroke. AJNR 2002;23:557-67. 